\title{
Influence from Imagining-Accuracy on Relaxation Effects during Imagining Music
}

\author{
Ryo Furukawa ${ }^{\mathrm{a}}$, Yusuke Mitani ${ }^{\mathrm{b}}$, Yuya Chiba ${ }^{\mathrm{c}}$, Yoshiko Maruyama ${ }^{\mathrm{a}}$, Kenji Moriya $^{\mathrm{a}^{*}}$, Masahiro Nakagawa ${ }^{\mathrm{d}}$ \\ aDepartment of Production Systems Engineering, National Institute of Technology (KOSEN), Hakodate College, \\ 14-1, Tokura-cho, Hakodate City, 042-8501, Japan \\ ${ }^{\mathrm{b}}$ Advanced Course of Production Systems Engineering, National Institute of Technology (KOSEN), \\ Hakodate College, 14-1, Tokura-cho, Hakodate City, 042-8501, Japan \\ 'Support Center for Engineering Education, National Institute of Technology (KOSEN), Hakodate College, \\ 14-1, Tokura-cho, Hakodate City, 042-8501, Japan \\ dDepartment of Electrical, Electronics and Information Engineering, Nagaoka University of Technology, \\ 1603-1, Kami-Tomioka-cho, Nagaoka City, 940-2137, Japan \\ *Corresponding Author: moriya@ hakodate-ct.ac.jp
}

\begin{abstract}
In our previous research, we found that listening to music was an effective method to rest during usual study breaks. In addition, similar relaxation as when listening to music was acquired by imagining music. However, this implied that the ease of imagining music was an important factor. Therefore, here we investigated how accurately music needs to be imagined in the brain to obtain the same relaxation effect as that while listening to music by measuring brain activity. The prefrontal activity was determined from changes in oxy- and deoxy-Hemoglobin (Hb) measured by 10-channel wearable optical topography. We found that almost the same relaxation can be obtained as that while listening to music by imagining music, with approximately $80 \%$ accuracy. Moreover, whether subjects knew the music or not was seemingly one of the most important factors. However, albeit subjects do not know the music, the relaxation would increase by repeating the imagination. In addition, some subjects became much more inactive by imagining music as if they were performing on the piano by themselves.
\end{abstract}

Keywords: Brain activity, Relaxation, Music.

\section{Introduction}

In general, human concentration lasts for approximately 45 minutes. If we lose concentration, errors tend to occur and work efficiency is likely decreased. A suitable rest between each task is assumed to be important to avoid the decrease of work efficiency. Listening to music represents an effective method for relaxing ${ }^{(1)}$. In our daily life, we sometimes "imagine" our favorite music in the brain. Our previous research showed that brain activity during imagining music became as low as during listening to music, when subjects used their favorite music in the experiment. Moreover, one of the most important factors was the ease of imagining music (2)(3). Therefore, we attempted to investigate how accurately music needs to be imagined in the brain to obtain the same relaxation as when listening to music, based on measurements of brain activity. From the signal measured by an optical topography, the complex functions such as feelings could be observed. Therefore, brain activity was determined by 10-channel-wearable optical topography.

\section{Experimental Methods}

\subsection{Experimental Protocol}

The experimental protocol is shown in Fig. 1. Subjects sat on a chair, kept their eyes opened during each task period and had their eyes closed during each relaxing period (listening music, imaging music, and just resting). The duration of each relaxing or task period was one minute. The sequence started with a resting period, the subjects then completed the 1-minute mental calculation 
task, then listened to the music, repeated the task, the rest, and the task. In order to reduce subjects' experimental physical burden, we designed total experimental time (i.e., time of wearing the instrument including preparation) within a $20 \mathrm{~min}$. Additionally, imagining unknown music for long term (e.g., over $2 \mathrm{~min}$ ) is very difficult task and could be heavy load for brain activities. Therefore, each task period was set as $1 \mathrm{~min}$. Because the subjects' blood flow as brain activity responds to task changes immediately, $1 \mathrm{~min}$ of the task period is not too short. Lastly, subjects imagined the music in the brain for one minute. Subjects repeated this protocol with the other music.

\subsection{Experimental Tasks}

Subjects were required to do mental calculations as tasks requiring concentration. Four mental calculation tasks were proposed: (I) addition of two two-digit numbers; (II) addition of three two-digit numbers; (III) addition of four two-digit numbers; or (IV) multiplication of two two-digit numbers. We observed the brain activity of each subject during the four types of mental calculations and chose a suitable level for each subject before each experiment.

Subjects were also given the following three type of breaks: (a) Listening to music, (b) imagining music, or (c) just resting. We prepared five kinds of classical music: (1) minor, (2) major, (3) monotonous, (4) complex, and (5) only piano. All classical music consists of only instrumental(s) without vocals. From these types of music, the subject selected (i) music known that the subject preferred, and (ii) music unknown that the subject liked the best and had not listened before. The two music were used for the experiment for the subject. In addition, two subjects did these experiments with the same music 5 weeks in a row in order to assess the influence of experience.

We let each subject intuitively answer the accuracy of their imagination of music using the Visual Analog Scale and defined this index as Imagining-Accuracy (IA) ${ }^{(3)}$.

\subsection{Brain Activity}

Figure 2 shows each measurement part and channel $(\mathrm{CH})$ of the frontal lobe. Brain activity was investigated by measuring changes in oxy- and deoxy-Hb density determined by 10-channel $(\mathrm{CH})$-wearable optical topography (WOT-100, Hitachi High Technologies Ltd.,) with Near-infrared spectroscopy ${ }^{(4-6)}$. If the density of oxy-Hb increases and that of deoxy-Hb decreases, it indicates that the area is active compared with the previous

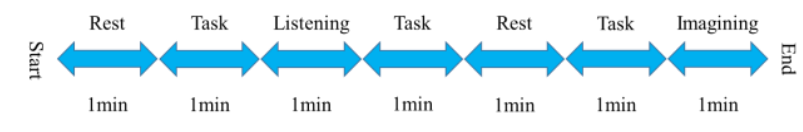

Fig. 1. Experimental protocol.

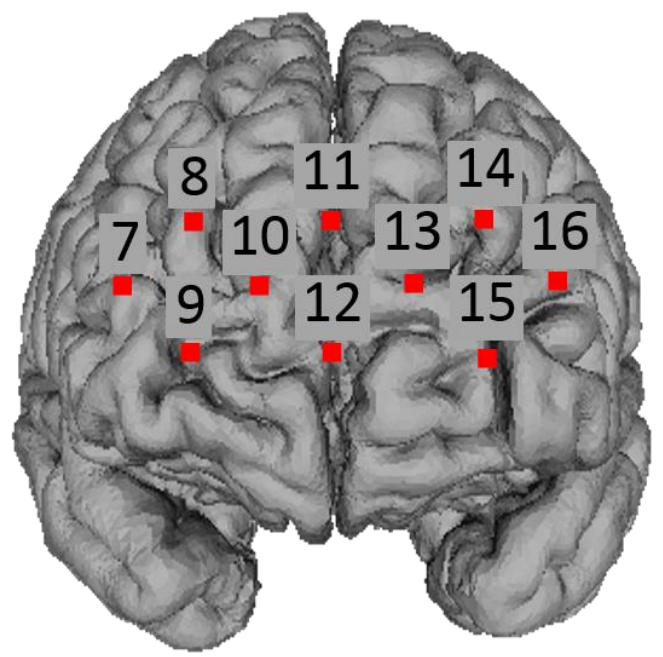

Fig. 2. Measurement $\mathrm{CHs}$

condition. On the contrary, when the density of oxy-Hb decreases and that of deoxy-Hb increases, there is no activity.

In this study, we analyzed the brain activity and the relaxation while resting in accordance with the following three procedures: (1) We compared the mean values of oxyand deoxy-Hb density during the task with the ones in the previous rest time, (2) We analyzed the tendency for the brain to become active or inactive from the variation of oxy- and deoxy-Hb density between each task and relaxing period, (3) We compared the mean values of oxy- and deoxy-Hb density when the brain had become inactive.

We excluded the first 10 seconds and the last 5 seconds from the analysis considering the influence of a sudden change of activity and retained 45 seconds for each 1-minute task period or resting period. The 45-seconds periods were divided into three sections (15 seconds each) and each mean value was calculated.

\section{Results and Discussion}

The experiment was conducted in 14 subjects (healthy students aged 16-20 years; eight males and six females). Two of these 14 subjects (Sub. 02 and Sub. 05) repeated the experiment 5 times with the interval of a week. Subjects repeated the experiment with the two music. Thus, 44 data were obtained through this experiment. Some data, which indicated active even while listening to music, were not 


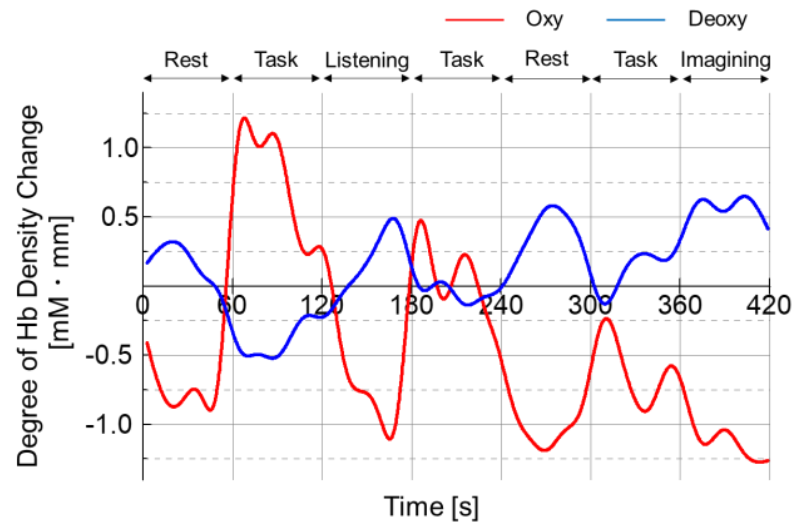

Fig. 3. Example of the measurement of $\mathrm{Hb}$ changes of

Sub. 05 (3rd week) through the "unknown music" sequence of the experimental protocol

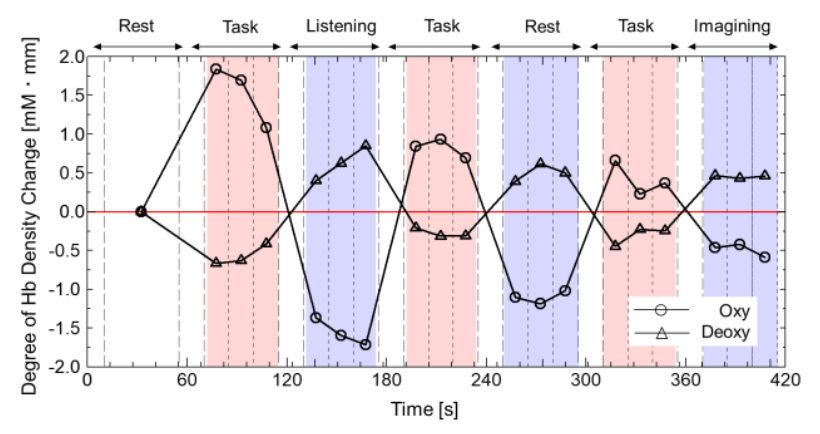

Fig. 4. Relative $\mathrm{Hb}$ change against mean value of previous task state calculated from Fig.3. Active period was indicated by light red area and inactive period was shown as light blue area respectively.

analyzed.

Figure 3 shows an example of the measurement of $\mathrm{Hb}$ changes in Sub. 05 (3rd week) through the "unknown music" sequence of the experimental protocol. The graph indicates the data of oxy- and deoxy-Hb density changes computed through wavelet transformation. The density of oxy-Hb increased and that of deoxy-Hb decreased in each task period. Thus, the brain tended to become active. On the other hand, the density of oxy-Hb decreased and that of deoxy-Hb increased in each relaxing period. Therefore, the brain tended to become inactive.

The $\mathrm{Hb}$ density changes compared with the mean values of $\mathrm{Hb}$ density in the previous activity (Fig. 4). We calculated and reported in the graph the average of each 15 -second period. The vertical axis indicates how much $\mathrm{Hb}$ density varied compared with that during the previous activity. We hypothesized that the more oxy-Hb decreases and the more deoxy-Hb increases, the lower brain activity.

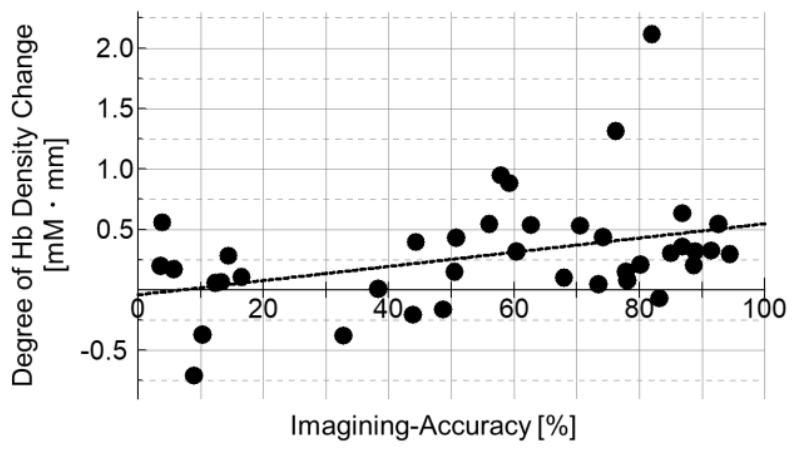

Fig. 5. The Hb density change and IA (all data)

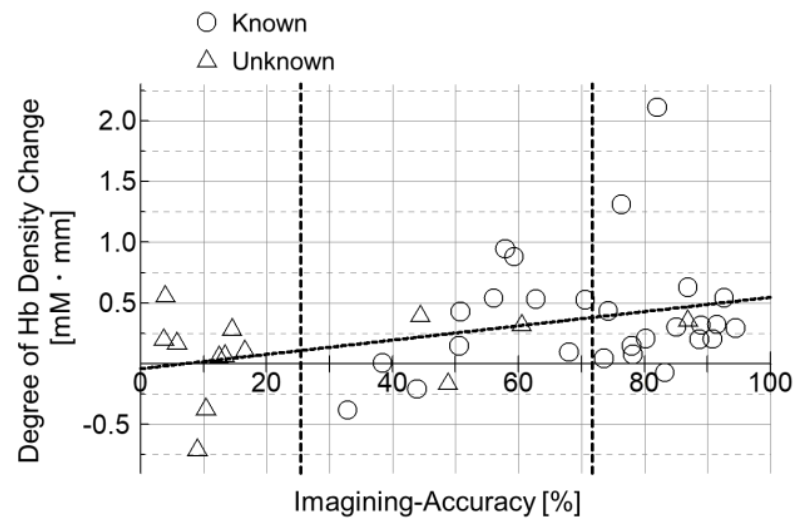

Fig. 6. The Hb density change and IA (according to two kinds of music)

The results of the degree of $\mathrm{Hb}$ density change and IA evaluated in this experiment (Fig.5). Here, the $\mathrm{Hb}$ density change indicates $\Delta$ Deoxy- $\Delta \mathrm{Oxy}$ defined as the degree of relaxation. Thus, the negative values correspond to high brain activity (brain was active). As IA increased, the $\mathrm{Hb}$ density change gradually increased and they were positively correlated: $|\mathrm{r}|=0.37$. Therefore, the more accurate the imagination was, the more the brain was inactive while imagining music.

The average IA of the "known music" was $71.7 \%$ and the IA of the "unknown music" was $25.4 \%$. Fig. 6 shows the results according to the two kinds of music. The data of each kind of music were distributed in the outside area of each average line. This result suggests that it is important to know the music for a higher level of relaxation approaching the level obtained while listening to music.

Figure 7 shows the influence of the experience impact of imagining music in the brain. While no variation was not observed for the "known music," both IA and $\mathrm{Hb}$ density change tended to increase for the "unknown music" the more experiment was repeated. Considering this result, the subjects learned the music and their relaxation increased by repeating the imagination. Moreover, the statistical 


\begin{tabular}{|llllll|}
\hline & $1^{\text {st }}$ & $2^{\text {nd }}$ & $3^{\text {rd }}$ & $4^{\text {th }}$ & $5^{\text {th }}$ \\
Sub. 02 & $\bullet$ & $\bigcirc$ & $\bigcirc$ & $\bullet$ & $\mathbf{0}$ \\
Sub. 05 & $\square$ & $\square$ & 口 & $\square$ & $\mathbf{\square}$ \\
\hline
\end{tabular}

(a)

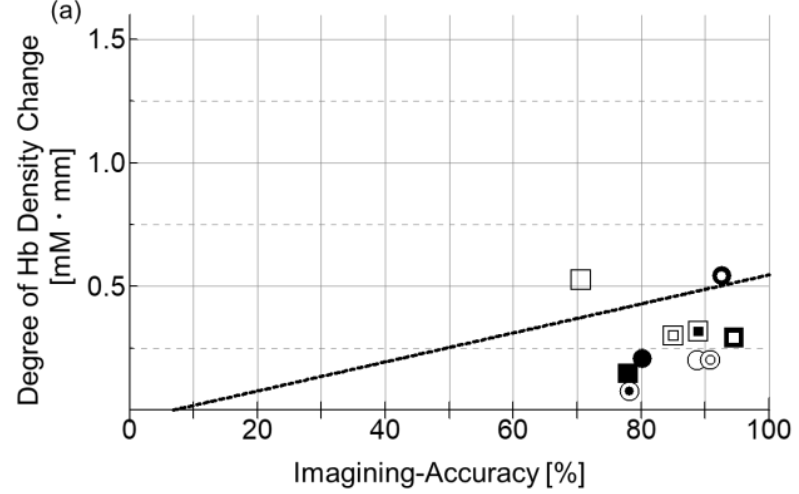

(b)

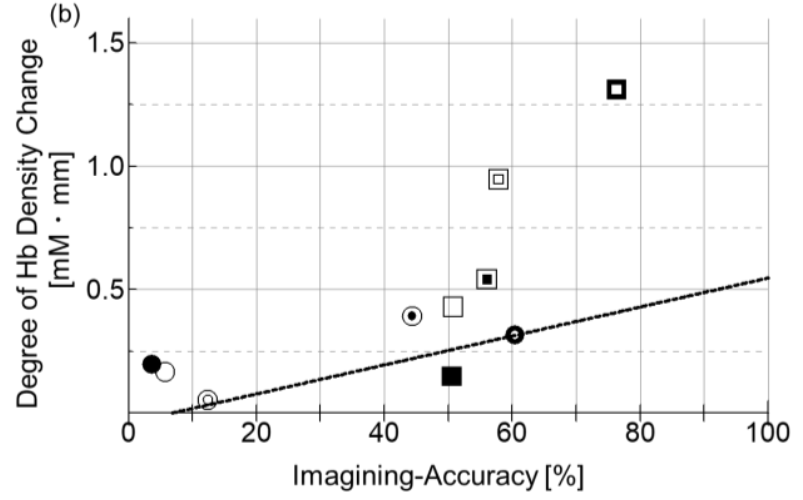

Fig. 7. The influence of experience of imagining music in the brain: (a) known; (b) unknown

difference between male and female was not significant ( $\mathrm{P}$ $>0.05$, ANOVA).

We focused on brain activity while imagining music compared with the brain activity while listening. Fig. 8 shows the rate of $\mathrm{Hb}$ density change and IA acquired in this experiment. The vertical axis indicates the rate of the $\mathrm{Hb}$ density change during Imagining to that during Listening to music: $\left(\Delta H b_{\text {Ima }} / \Delta H b_{\text {Lis }}\right) \cdot 100$ [\%]. Thus, in this figure, the negative values represent the brain becoming inactive while listening to music and active while imagining music. The correlation coefficient was 0.27 in Fig. 8 (a). The data point of Sub. 04 for the unknown music (*) was quite far from the regression line and represents an outlier. In fact, Sub. 04 became active even while imagining the known music. We presumed that Sub. 04 tended generally to be active while imagining music. Thus, we excluded the outlier data point from the data and obtained a correlation coefficient of 0.01 as shown in Fig. 8 (b). Moreover, the data which indicated active were 6 in total: 3 data of the known music; 3 data of the unknown music. It seemingly did not cause being active while imagining music to

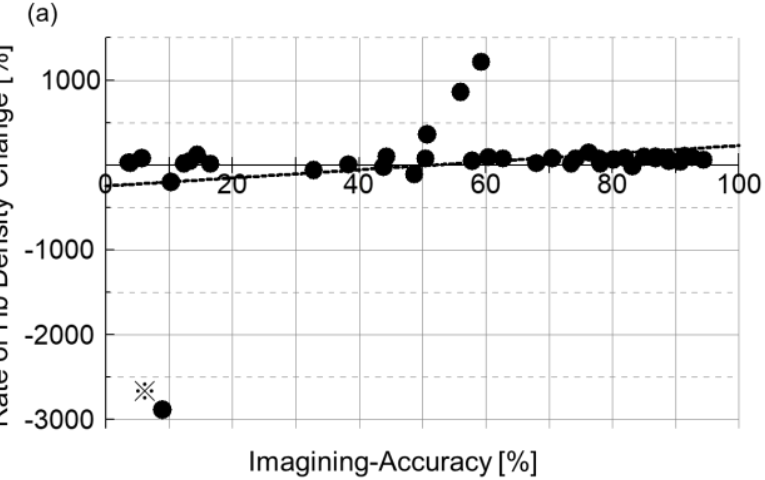

(b)

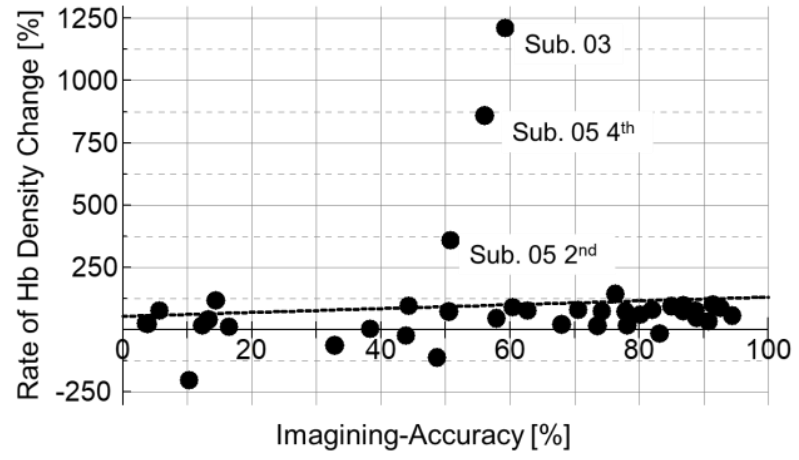

Fig. 8. The rate of the $\mathrm{Hb}$ density change while listening music to that while imagining music: (a) all data; (b) all data except the outlier *

imagine the unknown music.

The data of Sub. 03 and Sub. 05 well exceeded 100\%, which meant that they were much more relaxed while imagining music compared with while listening to music. Both of them were piano-experienced subjects. In addition, Sub. 05 had performed one music and Sub. 03 had performed both music on the piano before. This background affected the results and we discovered they tended to

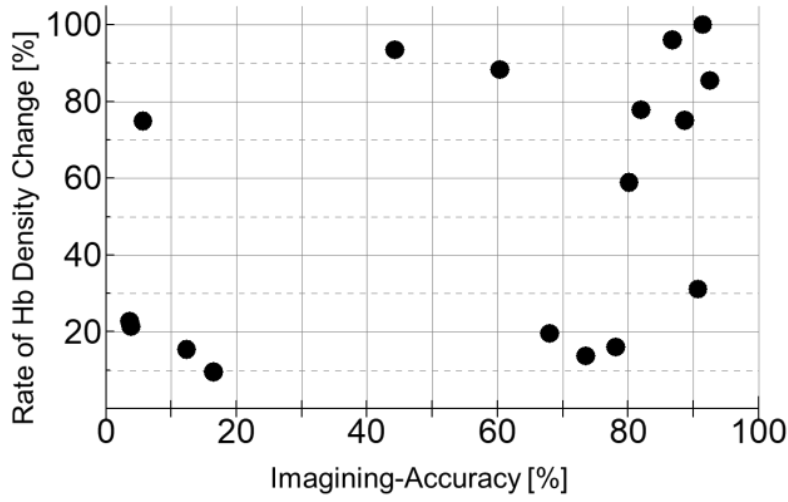

Fig. 9. The rate of the $\mathrm{Hb}$ density change while listening to music over while imagining (piano-experienced subjects excluded; only inactive) 
imagine music as if they were performing on the piano themselves. Thus, the most inactive brain activity occurred seemingly owing to imagining music as if they were performing on the piano themselves.

To investigate how accurately music needs to be imagined to obtain the same level of relaxation as while listening to music, we focused on the inactive data points of all subjects other than the piano-experienced subjects. Fig. 9 shows the general tendency of the rate of the Hb density change while listening over while imagining music. From this result, it is considered that we tend to acquire almost the same relaxation imagining music as that when listening to music with approximately $80 \%$ accuracy.

\section{Conclusions}

In this research, it was revealed that we tend to acquire almost the same relaxation level as that during listening to music by imagining music with approximately $80 \%$ accuracy. Moreover, whether subjects knew the music or not was seemingly one of the most important factors. However, albeit subjects do not know the music, the relaxation would increase by repeating the imagination. In addition, some subjects became much more inactive by imagining music as if they were performing on the piano themselves. Therefore, it was presumed that if we imagined in our own way, we could obtain much higher relaxation compared with one by imagining the recording.

\section{Acknowledgment}

A part of this study was supported by a grant from Nagaoka University of Technology for collaborative research with National Institute of Technology.

\section{Addition Statement}

This research was approved by the Life Ethics Committee of NIT, Hakodate College (KOSEN).

\section{References}

(1) Kenji Moriya, Ikusaburo Kurimoto, Nobuo Ezaki, and Masahiro Nakagawa: "Influences of Listening to Music in Study Break on Brain Activity and Parasympathetic Nervous System Activity", Journal of the Institute of Industrial Applications Engineers, Vol. 6, No. 1, pp. 34-38, 2018
(2) Yusuke Mitani, Takuya Kubo, Ikusaburo Kurimoto, Kenji Moriya: "Investigation of brain activity when listening to and imagining music", IIAE Annual Conference 2018, pp. 23-24, 2010 (in Japanese)

(3) Yusuke Mitani, Takuya Kubob, Yuy Chiba, Yoshiko Maruyama, Kenji Moriya, Masahiro Nakagawa: "Investigation of Brain Activity During Listening to and Imagining Music", Proceedings of 7th IIAE International Conference on Industrial Application Engineering 2019, pp. 4-8, 2019

(4) Shin Matsushita, Masahiro Nakagawa: "Emotional Information Analysis Using Optical Topography", Institute of Electronics, Information, and Communication Engineers A, Vol. J88 -A, No. 8, pp. 994-1001, 2005 (in Japanese)

(5) C. Gelinas, M. Boitor, M. Ranger, C.C. Johnston, M. Marchie, F. Cervero, M. Choiniere : "Is near infrared spec- ' troscopy valid for the detection of procedural pain in postoperative cardiac surgery intensive care unit adults?", Journal of Near Infrared Spectroscopy(online access), 2017

(6) S. Matsushita and M. Nakagawa : "Emotional Information Analysis using Optical Topography", IEIEC Trans. Fundamentals, Vol.J88-A, No.8, pp.994-1001, 2005 